\title{
C-band continuously tunable lasers using tunable fiber Bragg gratings
}

\author{
Shien-Kuei Liaw, ${ }^{\mathrm{a}}$, Kuan-Luen Hung ${ }^{\mathrm{a}}$, Yi-Tseng Lin ${ }^{\mathrm{a}}$, \\ Chia-Chin Chiang ${ }^{\mathrm{b}}$, Chow-Shing Shin ${ }^{\mathrm{c}}$ \\ ${ }^{a}$ Department of Electronic Engineering, National Taiwan University of Science and Technology, Number 43, Sector 4, Keelung Road, \\ Taipei 106, Taiwan, ROC \\ ${ }^{\mathrm{b}}$ Department of Mechanical Engineering, National Kaohsiung University of Applied Sciences, Kaohsiung 807, Taiwan, ROC \\ ${ }^{\mathrm{c}}$ Department of Mechanical Engineering, National Taiwan University, Number 1, Sector 4, Roosevelt Road, Taipei 106, Taiwan, ROC
}

Received 4 May 2006; received in revised form 5 August 2006; accepted 5 August 2006

Available online 28 September 2006

\begin{abstract}
We report the development of a ring tunable fiber laser based on tunable fiber Bragg gratings (TFBG) integrated with an optical circulator. The TFBG is embedded inside a 3-piont bending device for wavelength tuning. The tunable laser operating in the C-band has power variation, tuning resolution, tuning range and laser line width of $\pm 0.5 \mathrm{~dB}, 0.5 \mathrm{~nm}, 25.0 \mathrm{~nm}$ and less than $0.05 \mathrm{~nm}$, respectively. As $40 \mathrm{~mW}$ of pump power is used, the ring tunable laser has a side mode suppression ratio of $60 \mathrm{~dB}$ and a power conversion efficiency of $25 \%$. These specifications ensure the high-quality operation of a tunable laser.

(C) 2006 Elsevier Ltd. All rights reserved.
\end{abstract}

Keywords: Tunable laser; Fiber Bragg gratings; Fiber laser

\section{Introduction}

In recent years, lasers have found a variety of applications, including sensing, instrument testing, optical signal processing, optical communications, and photon analogto-digital conversion (ADC) [1,2]. More specifically, tunable lasers have been flexible for usage in wavelength routing, wavelength protection and optical measurement. Several methods have already been proposed to investigate tunable lasers using photonic crystal mirrors [3] and a multiple quantum-well waveguide. Among them, the use of the erbium-doped fiber ring laser (EDFRL) exhibits potential for success, partially since it features a lowtemperature sensitivity of wavelength. The selection of their operation wavelengths has been achieved by using different optical filtering devices, such as Mach-Zehnder filters or in-fiber comb filters. However, the tuning range can be narrow [4], or the line width can be wide due to large filter bandwidth. Another approach is the dual wavelength tunable fiber laser. Lasing occurs at two different

\footnotetext{
${ }^{*}$ Corresponding author. Tel.: + 886227376384 ; fax: + 88634812175

E-mail address: skliaw@et.ntust.edu.tw (S.-K. Liaw).
}

wavelengths, alternatively, by using a circuit consisting of two overlapping circuits with a common gain medium [5]. In this paper, we demonstrate the use of the tunable fiberBragg-gratings (TFBGs)-based tunable laser. A suitably designed optical device could cover the whole C-band region. The laser is constructed by a $1 \times 2$ optical switch (OSW) and optical circulator (OC). The results show that the tuning ability could be realized by appropriately switching the OSW and compressing/straining the TFBGs.

\section{Operation mechanism of the TFBG}

From the theory of solid mechanics [6], the transverse displacement $(v)$ is related to longitudinal strain $(\varepsilon)$ applied to the FBG for tuning purpose.

$E \varepsilon=\frac{M y}{I}$

and

$v=\frac{-P L^{3}}{48 E I}$.

In these equations, $E$ is the elastic modulus, $I$ is the moment of inertia, $M(=P L / 2)$ is the bending moment, 


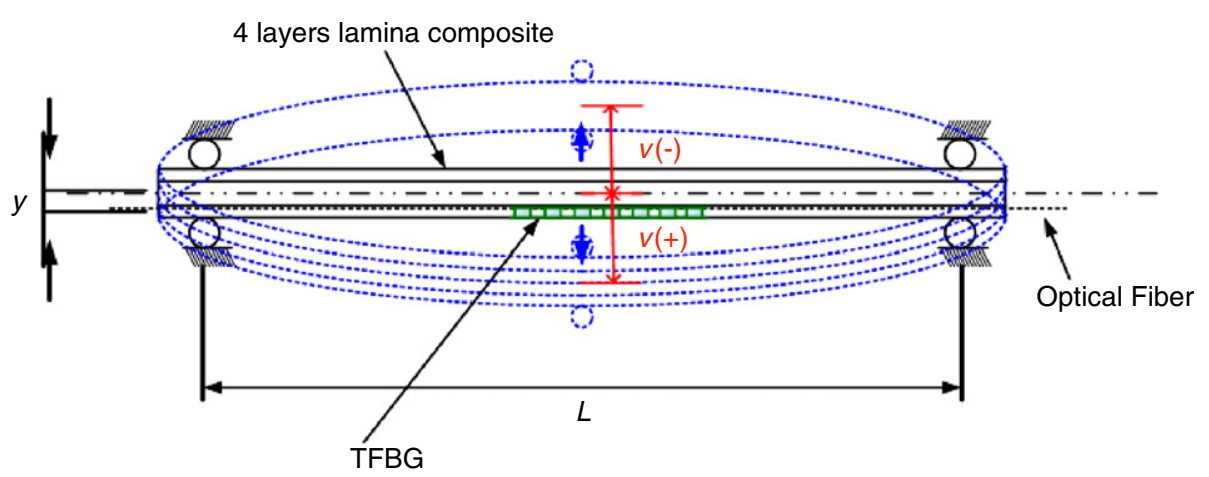

Fig. 1. Schematic diagram shows the concept of a 3-point bending device used for fiber Bragg grating tuning.

$P$ is the load, $L$ is the span of the 3-point bending support, and $y$ is the distance to the point at which the bending strain is desired, which is measured from the neutral axis. Thus, from Eqs (1) and (2), we realize the relationship between $v$ and $\varepsilon$ as

$\varepsilon=\frac{48 v y}{-2 L^{2}}$.

The wavelength shift is in linear proportion to strain [7] as

$\Delta \lambda_{\mathrm{B}}=\lambda_{\mathrm{B}}\left(K_{\varepsilon} \varepsilon+K_{\mathrm{T}} \Delta T\right)$,

here, $\lambda_{\mathrm{B}}$ is the peak wavelength of FBG, $K_{\varepsilon}$ is the strain constant, and $K_{\mathrm{T}}$ is the thermal constant. If the temperature is kept constant, the tuning wavelength $\left(\Delta \lambda_{\mathrm{B}}\right)$ is

$\Delta \lambda_{\mathrm{B}}=\lambda_{\mathrm{B}} \frac{48 K_{\varepsilon} v y}{-2 L^{2}}$

From Eq. (5), the tuning wavelength is proportional to the transverse displacement $(v)$.

By changing $v$, we are able to tune the TFBG for fiber laser application. First, we embed the FBG in the outer laminar as shown in Fig. 1. The composite with the TFBG embedded within is attached to a 3-point tuning device by using instant adhesive glue. Tuning, either by straining or compressing, the precision screw of the 3-point bending device, we can apply transverse displacement in either direction to easily attain a tunable range of $\pm 10 \mathrm{~nm}$. This eliminates any use of complicated or bulky components to perform the tuning function. Fig. 2 shows the superimposed tuning spectra of two homemade TFBGs. The demonstrated tuning range of each FBG is approximately $15 \mathrm{~nm}$ with reflectivity of $99.9 \%$. Before tuning, $\mathrm{FBG}_{1}$ has a central reflective wavelength of $1540.5 \mathrm{~nm}$ while that of $\mathrm{FBG}_{2}$ is $1552.7 \mathrm{~nm}$. We demonstrate the feasibility of $\mathrm{FBG}_{1}$ by tuning it from 1535.6 to $1545.5 \mathrm{~nm}$ and $\mathrm{FBG}_{2}$ by tuning it from 1547.7 to $1560.7 \mathrm{~nm}$. Fine tuning resolution as precise as $0.5 \mathrm{~nm}$ for $\mathrm{FBG}$ is realized.

\section{Configuration description of the TFBG laser}

Fig. 3 shows the proposed scheme of the wavelength tunable laser. This circuit includes $10 \mathrm{~m}$ of EDF, a

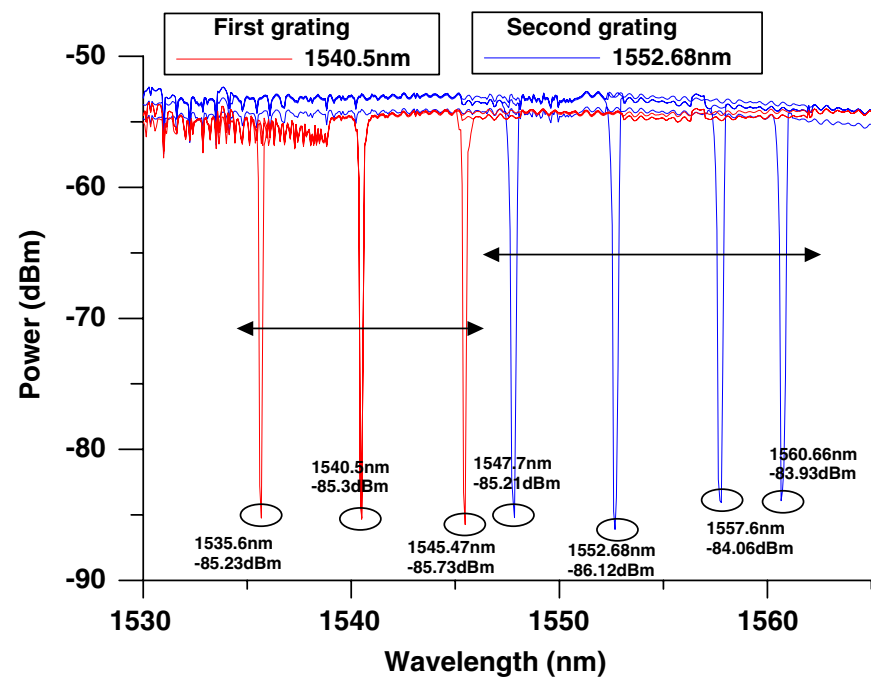

Fig. 2. Superimposed transmission spectra of two C band TFBGs, which have a precise tuning resolution of $0.5 \mathrm{~nm}$.

wavelength division multiplexing (WDM) coupler, and a $1480 \mathrm{~nm}$ pumping source operating at $40 \mathrm{~mW}$. The 3-port OC integrates with the FBGs, which act as reflective mirrors to form a looped-back circuit. On the right hand side, there is one $1 \times 2$ OSW and two TFBGs $\left(\right.$ TFBG $_{1}$, $\mathrm{TFBG}_{2}$ ) connected to either output port of the OSW. By compressing or straining these TFBGs, their overlapping spectra could cover the $\mathrm{C}$ band. The determined tuning wavelength, either the shorter $C$ band $(1530-1545 \mathrm{~nm})$ or the longer $\mathrm{C}$ band $(1546-1560 \mathrm{~nm})$, depends on the switching status of the OSW. The switching speed is $10 \mathrm{~ms}$ for this OSW. The transmission (or reflection) spectra are not degraded even if the TFBGs are tuned to the boundary points $(1530,1545$, or $1560 \mathrm{~nm})$. Note that the maximum tuning ability of the homemade TFBG is up to $\pm 10 \mathrm{~nm}$.

\section{Experimental results and discussion}

Fig. 4 shows superimposed optical spectra of the ring tunable laser with a side mode suppression ratio (SMSR) of $60 \mathrm{~dB}$. It could cover the C-band window using the 


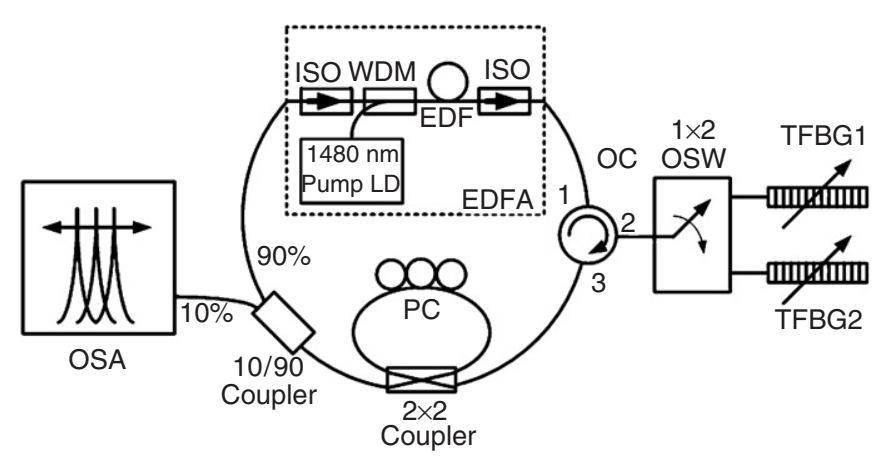

Fig. 3. TFBG-based ring tunable laser; PC: polarization controller; OSW: optical switch; OC: optical circulator; TFBG: tunable fiber Bragg grating; ISO: optical isolator; EDF: erbium-doped fiber; OSA: optical spectrum analyzer.

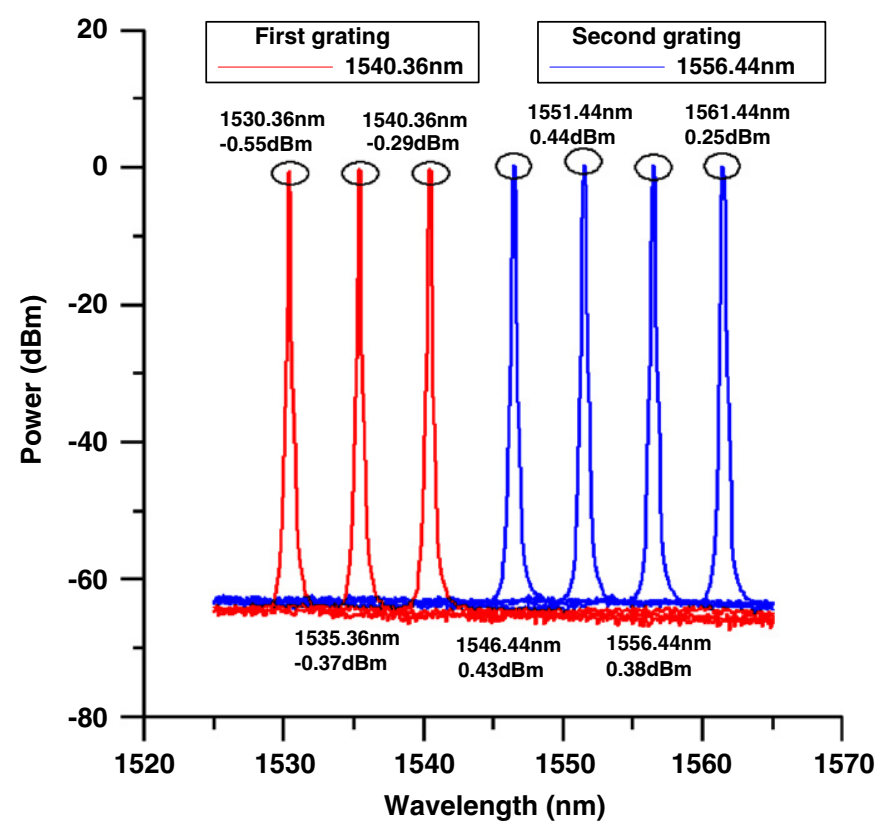

Fig. 4. Superimposed optical spectra of the ring tunable laser. The whole $\mathrm{C}$ band tuning is realized by using two TFBGs with different original wavelengths.

combined TFBGs, with tuning resolution of $0.5 \mathrm{~nm}$ for each TFBG. The output power increases as the reflectivity is increased. The average output power is $0.0 \mathrm{dBm}$ (i.e., $1 \mathrm{~mW}$ ) for each channel and power variation is less than $\pm 0.5 \mathrm{~dB}$ over the whole tuning range. Thus, no variable optical attenuator (VOA) is need for power equalization laser wavelength. The coupler ratio is $10 / 90$, with $90 \%$ of lasing power being looped back to the ring circuit and $10 \%$ of lasing power detected by an optical spectrum analyzer (OSA) or power meter. Since the pumping power of the $1480 \mathrm{~nm}$ laser diode is $40 \mathrm{~mW}$, the ring tunable laser has a pumping efficiency of $25 \%$.

The tunable laser proposed here is of a unidirectional ring circuit design. It is well suited for obtaining nearly single longitudinal mode operation due to the cancellation of spatial hole-burning effect in the traveling wave field of the ring circuit. Laser line width can be reduced by means of inserting sub-ring circuit(s) in the main ring circuit as in our prior work [8]. In this work, only a sub-ring circuit is included composed of a polarization controller (PC) and one 50:50 coupler. The main ring integrated sub-ring serves as a mode filter. The laser mode oscillates only at a frequency that simultaneously satisfies the resonant conditions of both the main circuit and the sub-ring circuit. The $\mathrm{PC}$ in the sub-ring circuit must be tuned to the same states of polarization as that of the main circuit to ensure that no polarization mode competition effect is observed. The free spectra range (FSR) of the ring circuit is defined as $\mathrm{FSR}=c / n L$. The lasing wavelength is set at $1533 \mathrm{~nm}$ by using one of the TFBGs and the mean refractive index of the optical fiber is about 1.5. The lengths of the main and the sub-ring circuits are close to 30 and $4.5 \mathrm{~m}$, corresponding to the FSRs of 6.6 and $44.0 \mathrm{MHz}$, respectively. Although an RF spectrum analyzer is not available for precise measurement of the laser line width, which is estimated to be much narrow than that of the resolution limitation of an OSA of $0.05 \mathrm{~nm}$.

A versatile and cost-effective laser source should have the tunable ability to allow one to choose the wavelength needed or to scan a range of bandwidth. Our proposed TFBG lasers are able to fulfill such requirements. In theory, the proposed method could be applied to the whole S, C and L bands by parallel connection of several TFBGs with a $1 \times \mathrm{N}$ OSW pair. It goes without saying that TFBGs with appropriate original central wavelengths are necessary.

\section{Conclusion}

We report the investigation of the ring tunable laser based on TFBG technology. Each TFBG is embedded inside a 3-point bending device for wavelength tuning. The power variation, tuning resolution, tuning range and laser line width are $0.5 \mathrm{~dB}, 0.5,25 \mathrm{~nm}$ and less than $0.05 \mathrm{~nm}$, respectively. The ring tunable laser also has $60 \mathrm{~dB}$ of SMSR to ensure high-quality operation. With the features mentioned above, the tunable laser is a potential candidate for high-speed modulation in either a digital or analog system. It may also find vast applications in optical communication and optical measurement.

\section{Acknowledgments}

The work is partially supported by NSC under projects No. 94-2219-E-011-007 and 94-2622-E-011-021-CC3, Taiwan, ROC. We thank Y.C. Lai, S.C Chen and C.R. Wang for their kind help.

\section{References}

[1] Liaw SK, Tseng HY, Chi S. Parallel pump-shared linear cavity laser array using $980-\mathrm{nm}$ pump reflectors or $\mathrm{N}$ pieces of gain fibers as selfequalizers. IEEE Photonic Technol Lett 2000;12(1):19-21.

[2] Liaw SK, Hung FC, Xu Z, Jiao Y, Qin T, Zhang H, et al. Wavelength tuning and multiple-wavelength array-waveguide-grating lasers. Opt Eng 2003;42:2178-9. 
[3] Chen HX, Babin F, Leblanc M, Schinn GW. Widely tunable singlefrequency erbium-doped fiber lasers. IEEE Photonic Technol Lett 2003;15(2):185-7.

[4] Chang SH, Hwang IK, Kim BY, Park HG. Widely tunable singlefrequency Er-doped fiber laser with long linear cavity. IEEE Photonic Technol Lett 2001;13(4):287-9.

[5] Xu YZ, Tam HY, Du WC, Demokan MS. Tunable dual-wavelengthswitching fiber grating laser. IEEE Photonic Technol Lett 1998;10(3) 334-6.
[6] Bickford William B. Mechanics of solid: concepts and applications. IRWIN 1994:491.

[7] Rao YJ. Recent process in applications of in-fibre Bragg grating sensors. Opt Lasers Eng 1999;31:297-324.

[8] Lee CC, Chen YK, Liaw SK. Single-longitudinal-mode fiber laser with passive multiple-ring cavity and its application for video transmission. Opt Lett 1998;23(5):358-60. 